\title{
Genetic analysis and population structure of wild and cultivated wishbone flower (Torenia fournieri Lind.) lines related to specific floral color
}

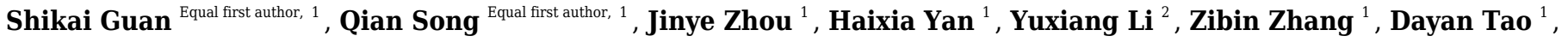 \\ Shuming Luo ${ }^{\text {Corresp., } 1,3}$, Youqiang Pan ${ }^{\text {Corresp. } 4}$ \\ 1 Flower Research Institute,Guangxi Academy of Agricultural Sciences, Nanning, Guangxi, China \\ 2 Guangxi Academy of Agricultural Sciences, Nanning, Guangxi, China \\ 3 Plant Breeding Institute, Faculty of Agriculture and Environment, University of Sydney, Sydney, Cobbitty, Australia \\ ${ }^{4}$ Guangxi Crop Genetic Improvement and Biotechnology Laboratory, Guangxi Academy of Agricultural Sciences, Nanning, Guangxi, China \\ Corresponding Authors: Shuming Luo, Youqiang Pan \\ Email address: shumingluo17@163.com, 347407647@qq.com
}

Background:The wishbone flower or Torenia fournieri Lind., an annual from tropical Indochina and southern China, is a popular ornamental annual plant, and many interspecific ( $T$. fournieri $\times T$. concolor) hybrid lines have been bred for the international market. The cultivated lines show a pattern of genetic similarity that correlates with floral color which informs on future breeding strategies. This study aimed to perform genetic analysis and population structure of cultivated hybrid lines comparing with closely related $T$. concolor wild populations.

Methods:We applied the retrotransposon based iPBS marker system for genotyping of a total of 136 accessions from 17 lines/populations of Torenia. These included 15 cultivated lines of three series: Duchess (A, B, C); Kauai (D, E, F, G, H, I, J); Little Kiss (K, L, M, N, P) and two wild T. concolor populations $(\mathrm{Q}$ and $\mathrm{R})$. PCR products from each individual were applied to estimate the genetic diversity and differentiation between lines/populations.

Results:Genotyping results showed a pattern of genetic variation differentiating the 17 lines/populations characterized by their specific floral colors. The final PCoA analysis, phylogenetic tree construction, and Bayesian population structural bar plot all showed a clear subdivision of lines/populations analysed. The 15 cultivated hybrid lines and the wild population Q that collected from a small area showed the lowest genetic variability while the other wild population $\mathrm{R}$ which sampled from a larger area had the highest genetic variability.

Discussion:The extremely low genetic variability of 15 cultivated lines indicated that individual line has similar reduction in diversity/heterozygosity from a bottleneck event, and each retained a similar (but different from each other) content of the wild genetic diversity. The genetic variance for the two wild $T$. concolor populations could be due to our varied sampling methods. The two wild populations $(\mathrm{Q}, \mathrm{R})$ and the cultivated hybrid lines (I, K, M, N, P) are genetically more closely related, but strong positive correlations presented in cultivated lines $A, C, E, M$, and $N$. These results could be used to guide future Torenia breeding.

Conclusions:The genetic variation and population structure found in our study showed that cultivated hybrid lines had similar reduction in diversity/heterozygosity from a bottleneck event and each line retained a similar (but different from each other) content of the wild genetic diversity, especially when 
strong phenotypic selection of floral color overlaps. Generally, environmental factors could induce transposon activation and generate genetic variability which enabled the acceleration of the evolutionary process of wild Torenia species. Our study revealed that wild Torenia populations sampled from broad geographic region represent stronger species strength with outstanding genetic diversity, but selective breeding targeting a specific floral color decreased such genetic variability. 


\section{Genetic analysis and population structure of wild and cultivated wishbone}

2 flower (Torenia fournieri Lind.) lines related to specific floral color

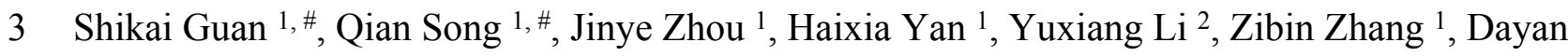

4 Tao ${ }^{1}$, Shuming Luo ${ }^{1,3, *}$ and Youqiang Pan ${ }^{4, *}$

$5 \quad{ }^{1}$ Flower Research Institute, Guangxi Academy of Agricultural Sciences, Nanning, Guangxi

6530007 , China

$7 \quad{ }^{2}$ Guangxi Academy of Agricultural Sciences, Nanning, Guangxi 530007, China

$8 \quad{ }^{3}$ Plant Breeding Institute, Faculty of Agriculture and Environment, University of Sydney, 107

9 Cobbitty Road, Cobbitty, NSW 2570, Australia

$10{ }^{4}$ Guangxi Crop Genetic Improvement and Biotechnology Laboratory, Guangxi Academy of

11 Agricultural Sciences, Nanning, Guangxi 530007, China

12 \# Shikai Guan, Qian Song contributed equally to this work

13 * Corresponding author at: Flower Research Institute, Guangxi Academy of Agricultural

14 Sciences, Nanning, Guangxi 530007, China. E-mail address: shumingluo17@163.com,

15 347407647@qq.com. 


\section{ABSTRACT}

Background: The wishbone flower or Torenia fournieri Lind., an annual from tropical Indochina and southern China, is a popular ornamental annual plant, and many interspecific ( $T$. fournieri $\times T$. concolor) hybrid lines have been bred for the international market. The cultivated lines show a pattern of genetic similarity that correlates with floral color which informs on future breeding strategies. This study aimed to perform genetic analysis and population structure of cultivated hybrid lines comparing with closely related $T$. concolor wild populations.

Methods: We applied the retrotransposon based iPBS marker system for genotyping of a total of 136 accessions from 17 lines/populations of Torenia. These included 15 cultivated lines of three series: Duchess (A, B, C); Kauai (D, E, F, G, H, I, J); Little Kiss (K, L, M, N, P) and two wild T. concolor populations ( $\mathrm{Q}$ and $\mathrm{R})$. PCR products from each individual were applied to estimate the genetic diversity and differentiation between lines/populations.

Results: Genotyping results showed a pattern of genetic variation differentiating the 17 lines/populations characterized by their specific floral colors. The final PCoA analysis, phylogenetic tree construction, and Bayesian population structural bar plot all showed a clear subdivision of lines/populations analysed. The 15 cultivated hybrid lines and the wild population $Q$ that collected from a small area showed the lowest genetic variability while the other wild population $\mathrm{R}$ which sampled from a larger area had the highest genetic variability.

Discussion: The extremely low genetic variability of 15 cultivated lines indicated that individual line has similar reduction in diversity/heterozygosity from a bottleneck event, and each retained a similar (but different from each other) content of the wild genetic diversity. The genetic variance for the two wild $T$. concolor populations could be due to our varied sampling methods. The two wild populations $(\mathrm{Q}, \mathrm{R})$ and the cultivated hybrid lines $(\mathrm{I}, \mathrm{K}, \mathrm{M}, \mathrm{N}, \mathrm{P})$ are genetically more closely related, but strong positive correlations presented in cultivated lines A, C, E, M, and N. These results could be used to guide future Torenia breeding.

Conclusions: The genetic variation and population structure found in our study showed that cultivated hybrid lines had similar reduction in diversity/heterozygosity from a bottleneck event and each line retained a similar (but different from each other) content of the wild genetic diversity, especially when strong phenotypic selection of floral color overlaps. Generally, environmental factors could induce transposon activation and generate genetic variability which enabled the acceleration of the evolutionary process of wild Torenia species. Our study revealed that wild Torenia populations sampled from broad geographic region represent stronger species strength with outstanding genetic diversity, but selective breeding targeting a specific floral color decreased such genetic variability.

\section{INTRODUCTION}

The genus Torenia belongs to the class Magnoliopsida, order Scrophulariales and family

Scrophulariaceae. There are more than 50 species native to tropical and subtropical Asia and 
53

54

55

Africa (Yamazaki, 1985; Hsieh and Yang, 2002). According to Yamazaki (1985), the significant biodiversity regions for this genus have been found in Southeast Asia (19 in Thailand, 20 in Cambodia, Laos, and Vietnam) and southern China (Hsieh and Yang, 2002). Wishbone flower or Torenia is the common name for several species in the genus including $T$. fournieri Lind., $T$. concolor Lindl., T. asiatica L. and T. hybrida (T. fournieri $\times$ T. concolor) (OGTR, 2008). T. fournieri, an annual from tropical Indochina, is one of the most important species in the genus for ornamental use in tropical and subtropical countries, especially as a bedding plant during summer in temperate regions. An erect type cultivar of this particular species with a violet floral color was initially commercialized. Then in 1988, PanAmerican Seed (Illinois, USA) released the Crown series with pink, white, and reddish-purple color lines. Selected from an interspecific cross between T. fournieri and T. concolor in 1995, a trailing type cultivar called the Summer Wave series was later released by Suntory Ltd. (Osaka, Japan). For more than 30 years, many hybrids have been released as patents or trademark series with a full range of floral colors from white with yellow throats to blue, cobalt, lavender, and violet, making Torenia a popular ornamental plant on the international market (Nagase, 1997; Miyazaki and Ohsumi, 1997; Tamura and Miyazaki, 1999; Miyazaki, 1999, 2001, 2002, 2003; Suzuki et al., 2002; Iwaki, 2005). As a consequence, a thorough knowledge of the genetic background of both wild and cultivated Torenia could aid future selective breeding of this important genus.

Early research employed $T$. fournieri as an ideal model plant for micromanipulation of artificial fertilization. Two major characteristics of the Torenia species included the exceptional protruding embryo sac (Higashiyama et al., 1997) and the synergid cells with polypeptides secreted as pollen tube attractants during fertilization (Okuda et al., 2009) that enable the detailed observation of the fertilization process. Consequently, the micromanipulation of artificial fertilization on Torenia as originally developed by Keijzer et al. (1988) resulted some interspecific hybrids, has since been adopted in a wide range of practical plant gene biotechnological experimentations (Higashiyama et al., 1998, 2001, 2006; Wu et al., 2008a, 2008b; Nakamura et al., 2010; Nishihara et al., 2018; Sasaki and Ohtsubo, 2020). However, the resulted interspecific hybrids require genetic analysis.

In relation to new variants, Tandon and Bhutani (1965) first reported the morphological and cytological descriptions of the colchicine-induced $T$. fournieri tetraploid plant, Kikuchi et al. (2005) later undertook cytogenetic analysis with the formation of Torenia interspecific hybrids. Kikuchi et al. (2007a) conducted a thorough evaluation of pollen tube growth in cross combinations between $T$. fournieri and fourteen related species and some interspecific hybrids were attained. Specifically on the T. fournieri and T. baillonii hybrid, a wide range of publications relating to cytological variations, genome size, interspecific genomic affinity, karyotypic variation, centromere separation and associations during mitosis or meiosis occurred (Kikuchi et al., 2006; 2007b; Nuntha et al., 2016; 2017). Wei (2010) completed some experimentation on inheritance of the floral color, and analyzed the barriers to distant hybridization between closely related species in the genus Torenia, whilst Liang (2014) outlined 
92 a practical method on selective and mutational breeding to further achieve interspecific polyploid

93 hybrids. The most recent interspecific Torenia hybrid was obtained through ovule culture

94 (Laojunta et al., 2019). However, there has been no population genetic analysis on interspecific

95 hybrids of Torenia.

96 A significant issue in the commercialization of Torenia interspecific hybrids is floral color, and

97 specific colors have been released as individual cultivars. Some early chemo-genetic

98 investigations of the floral color of T. fournieri revealed the inheritance pattern of anthocyanin

99 concentrations in their flowers, and similarly, the inheritance pattern of the carotenoids in the

100 petals of T. baillonii and T. fournieri have been described (Endo, 1962; Lang, 1970; Hess, 1971).

101 Suzuki et al. (2000) reported that hybrid floral color in Torenia could be modified by suppressing

102 the genes for anthocyanin biosynthesis whilst such floral color was later shown to be controlled,

103 expressed and modified by the flavonoid 3'-hydroxylase and flavone synthase II genes (Ueyama

104 et al., 2002). Through RNAi suppression of the anthocyanidin synthase gene, Nakamura et al.

105 (2006) achieved pure white flowers with a higher frequency and better stability than through the

106 antisense and sense suppression method. Furthermore, the floral pigmentation in Torenia species

107 could be mutated by heavy ion beam irradiation (Miyazaki et al., 2006). Additionally, Torenia

108 species exhibited a high degree of transformation by way of the Agrobacterium system (Aida and

109 Shibata, 1995), making them popular for transgenic studies (Aida et al., 2000a, 2000b; Aida,

110 2008; Nishihara et al., 2013; Masahiro et al., 2014). These molecular biotechnological research

111 results significantly enhanced our knowledge about floral color gene functioning in the Torenia

112 genus. Consequently, these research findings provide useful background information for the

113 future plant genetic improvement of Torenia floral color in cultivated and wild populations.

114 Regarding genetic activity and homeotic genes in particular, we know that they can regulate the

115 development of anatomical structures in various organisms during evolution. Bradley et al.

116 (1993) reported that some complementary floral homeotic phenotypes resulted from the opposite

117 orientations of a transposon at the plena locus. In addition, the first double flower phenotype in

118 Japanese morning glory (Ipomoea nil) resulted from gene duplication through insertion of an

$119 \mathrm{En} / \mathrm{Spm}$-related transposable element into a floral homeotic gene (Nitasaka, 2003). Similarly, the

120 chimeric TCP3 repressor in Arabidopsis was also found to be responsible for novel floral traits in

121 T. fournieri and Chrysanthemum morifolium (Narumi et al., 2011). For floral gene expression in

122 T. fournieri, the novel paracorollas trait has been generated when the floral homeotic genes were

123 induced by the plant hormone forchlorfenuron (Niki et al., 2012) because the relative cytokinin

124 biosynthesis system promoted a triggering expression of the cytokinin oxidase/dehydrogenase

125 gene during such treatments (Niki et al., 2013a; 2013b). Such research findings enable us to

126 conclude that some transposons in the homeotic genes can be the major genetic factors regulating

127 certain phenotypic traits of the flowers. Therefore, use of transposon marker systems could be

128 valuable in facilitating the population genetic analysis of Torenia in cultivated and wild

129 populations so as to understand their evolutionary history as well as the selective pressure due to

130 breeding for floral colors, in particular. 
131 Specifically, for a practical transposon marker system, Kalendar et al. (2010) designed the iPBS

132 (inter-Primer Binding Site) to deal with the "jumping genes" or transposable sequences of DNA

133 that move from one location to another in the genome. This molecular marker technology

134 utilized the universal presence of a tRNA complement as a reverse transcriptase primer binding

135 site in LTR (Long Terminal Repeat) retrotransposons (Alzohairy et al., 2014). Different to the

136 codominant marker systems such as microsatellite, iPBS is a dominant marker system that

137 requires no previous knowledge of the genome and it is a more powerful DNA genotyping

138 technique than the transposon-based markers established previously (Kalendar et al., 2019;

139 Androsiuk et al., 2019). This marker system has been successfully applied to a wide range of

140 plants such as ornamentals (Luo et al., 2015; Zhou et al., 2021), fruits (Baránek et al., 2012; Guo

141 et al., 2014; Mehmood et al., 2016; Al-Najm et al., 2016), medicinal plants (Chen and Liu, 2014;

142 Borna et al., 2017), and other different species (Singha et al., 2017; Androsiuk et al., 2019;

143 Androsiuk et al., 2020). Using such a retrotransposon-based iPBS technique, our objectives were

144 to investigate the genetic pattern, population structure and genetic relationships among 136

145 Torenia accessions including 15 cultivated lines from 3 commercial series (Duchess, Kauai,

146 Little Kiss) and two wild T. concolor populations sampled from two different sizes of area. Our

147 overall aim was: (i) to estimate the genetic variation and population structure for $T$. fournieri

148 cultivated lines which could be influenced by breeding selection; (ii) to explain the variation of

149 the genetic diversity between the two wild populations could be differed from the sampling

150 method we used; (iii) to provide insight into the effect on genetic variation in a population

151 experiencing a bottleneck in which strong phenotypic selection overlaps; (iv) to provide a

152 practical model for the genetic analysis of Torenia populations so as to better enable selective

153 breeding in the future.

\section{MATERIALS AND METHODS}

\section{Material}

156 The list of our 17 lines/populations included A (Duchess: Pink), B (Duchess: Burgundy), C

157 (Duchess: Deep Blue), D (Kauai: Burgundy), E (Kauai: Rose), F (Kauai: Deep Blue), G (Kauai:

158 Blue and White), H (Kauai: Magenta), I (Kauai: Lemon Drop), J (Kauai: White), K (Little Kiss:

159 White), L (Little Kiss: Burgundy), M (Little Kiss: Blue and White), N (Little Kiss: Rose

160 Picotee), P (Little Kiss: Blue), Q (wild: Lipu) and R (wild: Xichou). The origin of the plant

161 materials used in this study are listed in Supplemental File 1. We purchased a total of $15 T$.

162 hybrida seed lines from Shanghai Yuanyi Seedling Co. Ltd. These 15 colorful seed lines

163 included three commercial series. Two wild T. concolor populations (Q: Lipu and R: Xichou)

164 were collected from two different subtropical habitats in southern China, Lipu in Guangxi

165 Zhuangzu Autonomous Region and Xichou in Yunnan Province (Figure 1). To represent all the

166 commercial lines that have been produced by selective breeding and currently released on the

167 international market, approximately 200 seedlings for each line were germinated first, and eight

168 plants were randomly selected to form a line. According to the availability of individual $T$.

169 concolor plant in natural habitats, eight individual accessions of the population Q were collected

Peer) reviewing PDF | (2020:12:56689:2:0:NEW 28 May 2021) 
170 from a small area with a minimum distance of 50 meters, whilst population $\mathrm{R}$ was selected

171 across a larger area with a minimum distance of 100 meters. Commercial seeds were germinated

172 and grown in a glasshouse under a controlled environment at the Flower Research Institute,

173 Guangxi Academy of Agricultural Sciences in 2019. The floral colors for 17 populations were

174 presented in Figure 2. With a total of 136 accessions, 15 cultivated lines and two T. concolor

175 wild populations were then used for iPBS analysis.

\section{DNA extraction and quantification}

177 About $100 \mathrm{mg}$ of fresh young leaves from each accession were used for DNA isolation. Genomic 178 DNA for each plant was extracted using the EasyPure ${ }^{\mathrm{R}}$ Plant Genomic DNA Kit (TransGen

179 Biotech, Beijing, China) in accordance with the manufacturer's protocols. The quality and 180 quantity of DNA examined using $2.0 \%$ agarose gel electrophoresis by comparison with the 181 standard $\lambda$ DNA concentrations. A portion of the isolated DNA was diluted with distilled water 182 to $1 \mathrm{ng} \mu \mathrm{L}^{-1}$ concentration and used as the template for later PCR experimentation.

\section{Protocols for iPBS genotyping}

184 According to the sequence designed by Kalendar et al. (2010), the iPBS primers were 185 synthesized from Sangon Biotech (Shanghai, China) and DNA amplification in our study was 186 conducted using a slightly modified protocol. Using varied annealing temperatures, a total of 40 187 primers were initially screened with two DNA samples (A1, Q121), and the method was similar 188 to Zhou et al. (2012). Band pattern reproducibility of the PCR amplification was tested by 189 comparison with randomly selected electrophoretic profiles. To confirm band pattern 190 consistency, PCR was performed three times for each primer. Seven polymorphic primers with 191 repeatable and clearly identifiable bands were chosen for the final PCR amplifications (Table 1).

192 The PCR experiments were performed in $20 \mu \mathrm{L}$ reaction mixtures containing $1 \mu \mathrm{L}$ ( $1 \mathrm{ng}$ ) 193 genomic DNA, $10 \mu \mathrm{L} 2 \times$ Bench Top Taq Master Mix (Biomiga, San Diego, USA), $1 \mu \mathrm{L}$ of 10 $194 \mu \mathrm{M}$ primer (single primer), and $8 \mu \mathrm{L}$ of $\mathrm{dd}_{2} \mathrm{O}$. The PCR profile included an initial hot start at $19595^{\circ} \mathrm{C}$ for $3 \mathrm{~min}, 40$ cycles of denaturation at $94^{\circ} \mathrm{C}$ for $30 \mathrm{~s}$, annealing at $39-43.5^{\circ} \mathrm{C}$ for $30 \mathrm{~s}$ and 196 with an extension at $72^{\circ} \mathrm{C}$ for $2 \mathrm{~min}$. There was a final extension at $72^{\circ} \mathrm{C}$ for $5 \mathrm{~min}$ and the 197 program was terminated by holding at $4^{\circ} \mathrm{C}$. Using $0.2 \mathrm{~mL}$ tubes or $96-w e l l$ plates, all the 198 reactions was performed in a LIFE ECO Thermal Cycler (Bori, Hangzhou, China). Final PCR 199 products were electrophoresed at $70 \mathrm{~V}$ for 3.5 hours with $1.5 \%(\mathrm{w} / \mathrm{v})$ agarose gel in $1 \times \mathrm{TAE}$ 200 buffer (0.04 M Tris-acetate, 0.001 M EDTA). The standard Dongsheng LD DS5000 ladder 201 (Guangzhou, China) was used as the marker to estimate fragment lengths. GoldView I Nuclear 202 Acid Dye (10000×) (Suobao, Shanghai, China) was used to post-stain the gels for 20-30 min and

203 photographs were taken using the GenoSens 1860 model with Clinx GenoSens Capture Software 204 (Qinxiang, Shanghai, China).

205 Scoring and analyzing the iPBS data 
206 For the process of scoring, the DNA bands were carefully sized, visually examined and all faint

207 bands were excluded. Only clear bands were included and same sized bands were treated as a

208 single locus. The data record for each locus used ' 1 ' for presence of a band and ' 0 ' for absence

209 so as to form a binary matrix. The polymorphic informative content (PIC) value for each primer

210 was calculated by PIC $=1-\left[\mathrm{f}^{2}+(1-\mathrm{f})^{2}\right]$, where $\mathrm{f}$ is the frequency of the dominant marker in the

211 data set. Accountable bands consistently appeared in all the repeated experiments.

212 Accessions with different names that were completely matched at all the loci were considered as

213 duplicates. Duplicates in the data set were checked by multi-locus matching. All analyzes were

214 performed with the GenAIEx 6.5 program (Peakall and Smouse, 2012) (Figure 3). Pair-wise

215 genetic distance was first calculated using the DISTANCE procedure. Later analysis included

216 principal coordinates analysis (PCoA), and data were collected also as previously described in

217 Peakall and Smouse (2012). The individual based pairwise distance matrices for populations

218 were also used for hierarchical AMOVA analysis, and pairwise PhiPT values from the Phi-

219 statistics were used to measure population differentiation due to genetic structure estimated from

220 the genetic polymorphism data. Each time, the data were subjected to 1000 permutations. As an

221 indication of the relative contribution of population differentiation, the greater the pairwise

222 PhiPT values mean the greater the differences between populations. The total genetic variation

223 was partitioned across three levels: within populations (Phi-PT), among populations within taxa

224 (Phi-PR), and among taxa (Phi-RT).

225 For dendrogram construction, data were collected and used the MEGA7.0.14 software (Kumar et

226 al., 2016) for analysis as previously described in Zhou et al. (2021). There was a total of 148

227 positions for our 136 taxa in the final dataset. Using the UPGMA method (Sneath and Sokal,

228 1973), the optimal tree with the sum of branch length $=5.0639$ is shown. The final tree is drawn

229 to scale, with branch lengths in the same units as those of the evolutionary distances used to infer

230 the phylogenetic dendrogram. The evolutionary distances in the optimal tree were computed and

231 are in the units of the number of base substitutions per site (Tamura et al., 2004). A final

232 dendrogram revealing the evolutionary relationship of 136 Torenia taxa in 17 lines/populations is

233 listed in Figure 4.

234 The genotypic data were also analyzed using a Bayesian model-based clustering method 235 implemented through STRUCTURE V2.3.4 (Pritchard et al., 2000) for population structure to

236 classify 17 Torenia lines/populations into different groups. Data were collected as previously 237 described in Zhou et al. (2021). In Figure 5, the optimal number of clusters or the most likely K 238 value $(\mathrm{K}=5)$ was based on Evanno et al. (2005). Population structures were constructed using a 239 Bayesian model-based clustering computation from STRUCTURE HARVESTER (Earl and 240 Vonholdt, 2012), and a final population structure was optimized in Figure 6.

\section{RESULTS}

\section{Genetic DNA polymorphism}


243 We screened 7 primers with high polymorphism from 40 iPBS primers. These seven primers

244 (2076, 2077, 2272, 2277, 2377, 2387 and 2383) were subsequently selected for the final PCR

245 amplifications (Table 1). The sizes of scorable and reproducible bands from all these seven

246 primers ranged from 150 to $4500 \mathrm{bp}$. The number of unique banding patterns among these 136

247 accessions validated the use of this retrotransposon based iPBS marker system for individual

248 identification of the 17 lines/populations tested.

249 A total of 148 scorable bands were amplified using the seven primers selected, and they revealed

250 a high degree of genetic variability within each lines/population and among lines/populations.

251 Detailed results for these seven primers including the sequence of the primer, the annealing

252 temperature used in the PCR, number of amplified bands, number of bands specific for three of

253 the commercial series (Duchess, Kauai, Little Kiss) and wild populations, and mean PIC values

254 are presented in Table 1. The highest number of bands (28) was produced by primer 2277 while

255 primer 2377 generated the lowest (16). Primer 2277 had the highest PIC value (0.327) whereas

256 primer 2077 had the lowest (0.220). The mean PIC value for these seven primers was 0.267 . For

257 dominant markers, the PIC values varied between 0 to 0.5 , and any values more than 0.25 were

258 classified as highly informative. These results indicate that a wide range of genomic DNA

259 diversity existed across the 17 Torenia lines/populations tested.

260 Our results also showed that a range of different private bands were specifically amplified for

261 lines/populations with varied floral colors. As for the two wild populations, population Q shared

262 more common bands with the 15 cultivated lines, while population $\mathrm{R}$ had the highest number of

263 specific bands. For the 15 cultivated lines, there were a total of 152 population-specific bands

264 from seven primers. Primer 2076 represented 33 specific bands (in red and blue). Primer 2277

265 showed a maximum of 36 while primer 2077 had a minimum of six (Supplemental File 5).

\section{Heterozygosity for 17 Torenia lines/populations}

267 Summary statistics including number of different alleles, number of effective alleles, Shannon's

268 information index, expected heterozygosity, unbiased expected heterozygosity, and percentage of

269 the polymorphic loci are presented in Table 2. The number of different alleles $(\mathrm{Na})$ ranged from

$2700.4865(\mathrm{~J})$ to $0.9932(\mathrm{R})$, with an average of 0.6586 . The number of effective alleles $(\mathrm{Ne})$ varied

271 from $1.0243(\mathrm{~J})$ to $1.2885(\mathrm{R})$, with an average of 1.0899 . The Shannon's information index for

272 the 17 lines/populations ranged from $0.0200(\mathrm{~J})$ to $0.2453(\mathrm{R})$, with an average of 0.0758 . The

273 expected heterozygosity values (He) fluctuated from $0.0138(\mathrm{~J})$ to $0.1655(\mathrm{R})$, with an average of

274 0.0513. The unbiased expected heterozygosity $(\mathrm{uHe})$ ranged from $0.0147(\mathrm{~J})$ to $0.1766(\mathrm{R})$ with

275 an average of 0.0547 whereas the percentage of polymorphic loci ranged from $3.38 \%(\mathrm{~J})$ to

$27645.27 \%(\mathrm{R})$. Therefore, the most variation of genetic diversity existed between the cultivated line

$277 \mathrm{~J}$ and the wild population $\mathrm{R}$.

278 In Table 3, pairwise population PhiPT values for a total of 136 accessions from 17

279 lines/populations are listed. The greatest pairwise population PhiPT values are indicated in bold,

280 and their values varied from $\mathrm{N} / \mathrm{I}(0.917)$ to $\mathrm{Q} / \mathrm{I}(0.898)$. These results indicate that cultivated line 
$281 \mathrm{~N}$ and wild population Q are considerably different or varied compared to lines C, I and J. In

282 addition, the smallest pairwise population PhiPT values are marked in red and these values were

283 separated in two clusters. The first group contained M/P (0.336), N/P (0.562), M/N (0.588),

284 indicating less differentiation among populations $\mathrm{M}, \mathrm{N}$, and $\mathrm{P}$. The second group included $\mathrm{A} / \mathrm{E}$

285 (0.542), A/F (0.563), $\mathrm{F} / \mathrm{E}(0.581)$, and these results showed relatively close genetic relationships

286 among populations A, E, and F. Comparing all the 15 cultivated lines with the two wild $T$.

287 concolor populations in Supplemental File 2, the generalized genetic distance, and relationships

288 within and among populations outlined an overall population genetic evolutionary history. Our

289 AMOVA analysis further showed that most of the described genetic variation for 17

290 lines/populations occurred among lines/populations (79\%), and the variation within

291 lines/populations accounted for the remaining $21 \%$. However, the variation among 15 cultivated

292 lines was $14 \%$, and the variation within lines was $86 \%$ (Table 4 ).

293 Principal coordinates analysis (PCoA) for line/population correlations

294 Based on all the Euclidean distances between all individual accessions from our 17 Torenia

295 line/populations, principal coordinates analysis (PCoA) showed that the value of the first three

296 PCoA axes accounted for a total variation of $45.03 \%$ (first axis $=22.22 \%$, second $=12.26 \%$,

297 third $=10.56 \%$ ) (Figure 3). The spatial demonstration of the relative genetic distances revealed

298 the pattern of distribution of all the 136 accessions according to their genetic components or

299 background. Projection of the analyzed lines/populations on the first two axes showed that the

300 accessions of lines A, B, C, D, E, F were mainly clustered on the top right plane whilst the

301 accessions of lines $\mathrm{G}, \mathrm{H}, \mathrm{I}, \mathrm{J}, \mathrm{K}$, and $\mathrm{L}$ were distributed on the lower right plane. One sub-group

302 including lines $\mathrm{M}, \mathrm{N}, \mathrm{P}$ together with another sub-group of the wild population Q were scattered

303 on the upper left plane. The wild population $\mathrm{R}$ was scattered on the lower left plane as the only

304 single population registered there. Among this overall pattern of distribution, accessions of each

305 line/population tended to be clustered together closely, but the position of the accessions for

306 several commercial lines overlapped with a certain percentage.

307 In relation to the PCoA analysis, a total of 148 loci were combined into eight components as

308 eigen values by axis in relation to the sample eigen vectors and the list of these results are

309 presented in Supplemental File 3. The first component, which accounted for $51.49 \%$ of the total

310 variability, included lines A, C, E with high eigen values (marked in red), and lines/populations

$311 \mathrm{R}, \mathrm{Q}, \mathrm{N}$ with negative eigen values (marked in blue). Thus, lines A, C, E had a high positive

312 correlation with the highest loading factors whilst populations R, Q, N had a high negative

313 correlation with the lowest loading factors. Similarly, for the second component which accounted

314 for $28.40 \%$ of the whole variability, lines E, M, N (marked in red) were positively correlated

315 with higher loading factors, but lines R, I, K (marked in blue) were negatively correlated with

316 lower loading factors. The third component accounted for $26.20 \%$ of the variability, and lines R,

317 Q, D were positively correlated with higher loading factors whilst K, M, P were negatively

318 correlated with lower loading factors. All the other components were interpreted similarly, and a 
319 combined result from all the eight components could be formed to reveal the overall genetic

320 correlation relationships of the 17 Torenia lines tested.

321 Phylogenetic relationships for 17 Torenia lines/populations

322 Apart from the above principal coordinates analysis (PCoA), the evolutionary genetic

323 relationship of our 136 accessions was also drawn from the combined data from seven iPBS

324 primers (see above) using MEGA7 software (Fig. 3). The phylogenetic dendrogram was

325 constructed to scale with the branch lengths matched to reveal the evolutionary distances. All the

326136 accessions from the 17 lines/populations were placed into five major clusters in the final

327 optimal tree (Fig. 4, Supplemental File 4). The first cluster included all the 48 accessions from

328 six lines/populations A, B, C, D, E and F. The second group included 48 accessions of six lines

$329 \mathrm{G}, \mathrm{H}, \mathrm{L}, \mathrm{I}, \mathrm{J}$ and $\mathrm{K}$. The third group included all of the 24 accessions in lines $\mathrm{M}, \mathrm{N}$ and $\mathrm{P}$. The

330 fourth cluster included all the eight accessions of the wild population Q. The fifth cluster

331 contained all of the eight accessions of the wild population R. Consequently, each of the first two

332 major clusters in the dendrogram contained several sub-groups. It is noteworthy that all of the

333136 accessions from the 17 lines/populations were clearly grouped according to the origin of the

334 line/population with reference to their specific floral color (Supplemental File 4).

335 Population genetic structure and differentiations

336 Population stratification of the accessions was based on the most probable or maximum number

337 of $\mathrm{K}$ because $\Delta \mathrm{K}$ is the potential number of genetic clusters that may exist in the overall sample of

338 individuals examined. To calculate the value of $\Delta \mathrm{K}$ and the second-order rate of change of the

339 likelihood function of $\mathrm{K}$ and $\mathrm{m}\left(\left|\mathrm{L}^{\prime \prime}(\mathrm{K})\right|\right) / \mathrm{sd}[\mathrm{L}(\mathrm{K})]$, the optimal number of genetic clusters for these

340136 accessions was determined to be five $(\mathrm{Kopt}=5$, Figure 5). Consequently, the optimal number

341 of clusters of the uppermost hierarchical level of the population structure was achieved based on

$342 \mathrm{~K}=5$ (Figure 6). The final genetic structure showed significant differentiation with a continuous

343 linear order of the 136 accessions of the 17 lines/populations with their specific floral color. Within

344 the final bar plot, each colored bar represents the most likely ancestry of the cluster for the whole

345 or part of the genotype derived, and each vertical line represents an individual multi-locus

346 genotype. Thus, multiple-colored individual accessions have an admixture of genotype

347 components from multiple clusters or groups. Results from these overall structural partitions

348 agreed significantly with the distribution pattern plotted in the principal coordinate analysis

349 mentioned above (Figure 3), and the evolutionary phylogenetic relationships outlined in the

350 dendrogram (Figure 4).

\section{DISCUSSION}

352 A collection of 136 accessions of 15 cultivated lines and two wild $T$. concolor populations with

353 specific floral color were investigated using seven retrotransposon-based iPBS primers, and our

354 results are presented as a PCoA plot, a phylogenetic tree, and an optimized population genetic

355 structure bar plot. All the 148 loci generated from 7 selected primers were sufficiently powerful 
356 to discriminate genetically our individual accessions into the origin of the line/population, whilst

357 indicating an obvious pattern of genetic relationships within and among the lines/populations.

358 Results from these analyses, individually and combined, indicate that some morphological traits

359 such as the floral colors could be used as an associated genotypic identification, thereby

360 potentially guiding breeding practices that target such traits. This research also showed that the

361 comparison of cultivated T. fournieri lines and T. concolor wild populations are informative, and

362 these two different species are being compared when analyzing the results.

363 We know that population structure analyses using dominant markers such as the iPBS system can be weakened if the heterozygosity remains undetected or underestimated. To prevent such an issue in our analysis, we adopted $P h i$-statistics which are a modified version of the conventional Wright's $F$-statistics. Taking into consideration the genealogical information from the molecular data, the pairwise population PhiPT values were applied to measure the reduction of the expected level of heterozygosity in the population compared to a Hardy-Weinberg expectation that is usually used. Specifically, the Phi-statistics analysis we used showed the correlation of some genes or loci within the subdivided lines/populations. In particular, the genetic pattern and structure of the wild population Q (selected within a relatively small area) was similar to the 15 cultivated lines, but was significantly different from the other wild population $\mathrm{R}$ (selected across a much larger area). This most likely indicates that these differences in genetic diversity were resulted from bottleneck/drift effects when bringing a small number of individuals into cultivation during breeding practices. Thus, this Phi-statistics method could be used further to compare the genotype data of the parent lines against the hybrid lines so as to estimate such bottleneck/drift effects mentioned above. On the other hand, it needs to be noted that when considering wild populations within their natural habitats, sampling methods are one of the major factors affecting the conclusions being drawn about the population genetic structure.

Since blue is the common wild type of floral color for T. fournieri and T. conclor, and our two wild $T$. concolor populations had blue flowers, all the cultivated lines were bred for a specific floral color. In our study, clustering information among cultivated lines and wild populations via our PCoA analysis showed interesting patterns, and much of the variance in genotype among lines is due to differences between certain sets of lines which are most distant from one another. For instance, among cultivated lines I, K, M, N, P and the two wild populations Q and R, there were similar reduction in diversity/heterozygosity from a bottleneck event, and each line/population will retain a similar (but different from each other) content of the wild genetic diversity. As Mehmood et al. (2014) has shown, it is highly probable that the relevant floral color of these lines/populations is associated with a negatively correlated genetic relationship with the wild genetic content mentioned above. Therefore, as the highest loadings in the first two components, strong positive correlations presented in lines A, C, E, M, N, and these cultivated lines have less content of the wild genetic diversity. Consequently, these results from our population genetic analyses could guide future Torenia breeding. 
394 Being useful for reconstructing the evolutionary history of lines/populations from population 395 genetic analysis, the population genetic distance can be interpreted as a measure of genetic 396 divergence between species or between lines/populations within a species, whether the distance 397 evaluate the time from common ancestor or the degree of differentiation. Generally, species or 398 wild populations of a species with many similar alleles exhibit small genetic distances due to having had a recent common ancestor. Results from our study indicated that the wild population Q is genetically more closely related to lines M, N, P and they are likely therefore to have had an overall genetic similarity. For the purpose of expanding heterozygosity, these closely related lines/populations should not be used as parent candidates for any inter-line/population crosses, especially those targeting introgressive traits from wild populations. On the contrary, it would be better to cross these closely related lines/populations with other lines/populations containing more genetic differentiation such as A, B, C, D, E and F.

406

407

408

409

410

411

412

413

414

415

416

417

418

419

420

421

422

423

424

425

426

427

428

429

430

431

432

As previously indicated, this study revealed an extensive genetic diversity and structural pattern within and among the 17 Torenia lines/populations studied through the 136 accessions. A large number of specific bands deserve special attention as they were private alleles specific to the line(s)/population(s). The genetic information from these specific alleles indicated significant genetic relationships that separated certain lines/populations, as presented in the above PCoA analysis, phylogenetic dendrogram, and the final population structural bar plot. Furthermore, such population specific polymorphic bands can be excised, then purified from the gel and sequenced to provide more thorough genomic information relevant to the genetic analysis of the population. These specific molecular markers could also be associated with particular phenotypic traits of interest, such as floral color.

Regarding the heterozygosity for 17 Torenia populations tested, 15 commercial lines and the wild population $\mathrm{Q}$ had low values of $\mathrm{Na}$ (number of different alleles), $\mathrm{Ne}$ (the number of effective alleles), I (Shannon's information index), He (expected heterozygosity), and uHe (unbiased expected heterozygosity). Line $\mathrm{J}$ has the lowest value of records whilst wild population $\mathrm{R}$ had the highest values. Since wild population $\mathrm{Q}$ was sampled from a smaller area, and population $\mathrm{R}$ was sampled across a much larger area, the different results of these two wild populations could be due to our sampling practices, as suggested above. Furthermore, the mean value of I (0.0758), He (0.0513) and uHe (0.0547) of all the 17 Torenia populations tested are considerably less than that reported previously in other genera of plants by Gailite and Rungis (2012), Guo et al. (2014), Baránek et al. (2012), Mehmood et al. (2016), Al-najm et al. (2016) and Borna et al. (2017). Our lower values indicate significant low genetic diversity/heterozygosity in the 15 Torenia series that have been released, and in the wild population Q. This suggests either some kind of bottleneck/drift effect by selection during breeding practices.

Our results also support the view that the retrotransposon-based iPBS system we used could also be used to estimate genetic diversity and structure of other plant species. This would enable population genetic analysis on other biological phenomena such as adaptation, speciation, and

Peer) reviewing PDF | (2020:12:56689:2:0:NEW 28 May 2021) 
433 population structure. Whilst Torenia species have been used previously for frontier research on 434 fertilization and gene engineering of ornamental characteristics within horticultural ecosystems, 435 our research conclusions could complement and expand its use as a model plant for new interests 436 in plant population genetic analysis. Therefore, the results from this study could have two main 437 flow-on effects. The first could be information applicable to selective breeding for commercial 438 purposes. A second application could be with endangered species because the population genetic 439 analysis would be able to index the strength of the population of the species in its natural habitat.

\section{CONCLUSIONS}

441 The iPBS markers showed interesting patterns of genetic variation among wild $T$. concolor 442 populations and T. fournieri cultivated lines with different floral color. For cultivated lines and 443 wild populations, there were similar reduction in diversity/heterozygosity from a bottleneck 444 event, and each line/population retained a similar (but different from each other) content of the 445 wild genetic diversity. Variances in genetic diversity/heterozygosity for the two wild populations 446 could be explained by differences in sampling method. Environmental factors could induce 447 transposon activation and generate genetic variability which enabled the acceleration of the 448 evolutionary process of wild Torenia species, but selective breeding targeting a specific floral 449 color decreased genetic diversity. As a consequence, this study also provide insight into the 450 effect on genetic variation in a line/population experiencing a bottleneck in which strong 451 phenotypic selection overlaps, but wild Torenia populations sampled from broad geographic 452 region represent stronger species strength with outstanding genetic diversity. Using of the iPBS 453 marker system not only revealed genetic variation and population structure for wild and 454 cultivated Torenia plants, but this result could guide future selective breeding especially for 455 commercial purposes.

\section{Acknowledgements}

457 We express our gratitude to the Rice Research Institute and the Horticultural Research Institute, 458 Guangxi Academy of Agricultural Sciences for providing some of the facilities for this research.

459 The authors would like to thank the Academic Editor (Dr. Ruslan Kalendar) and reviewers for 460 their most helpful comments and suggestions.

\section{REFERENCES}

462 Aida R, Shibata M. 1995. Agrobacterium-mediated transformation of Torenia (Torenia 463 fournieri). Breeding Science 45(1): 71-74.

464 Aida R. 2008. Torenia fournieri (torenia) as a model plant for transgenic studies. Plant 465 Biotechnology 25(6): 541-545.

466 Aida R, Yoshida K, Kondo T, Kishimoto S, Shibata M. 2000a. Co-pigmentation gives bluer 467 flowers on transgenic torenia plants with the antisense dihydroflavnonol-4-reductase gene. Plant 468 Science 160: 49-56. 
469 Aida R, Kishimoto S, Tanaka Y, Shibata M. 2000b. Modification of flower color in torenia 470 (Torenia fournieri Lind.) by genetic transformation. Plant Science 153: 33-42.

471 Al-Najm A, Luo S, Ahmad N, Trathwan R. 2016. Molecular variability and genetic relationships 472 of date palm (Phoenix dactylifera L.) cultivars based on inter-primer binding site (iPBS)

473 markers. Australian Journal of Crop Science 10(5): 732-740.

474 Alzohairy AM, Gyulai G, Ramadan MF, Edris S, Sabir JSM, Jansen RK, Eissa HF, Bahieldin A. 475 2014. Retrotransposon based molecular makers for assessment of genomic diversity. Functional 476 Plant Biology 41: 781-789.

477 Androsiuk P, Koc J, Chwedorzewska KJ, Gorecki R, Gielwanowska I. 2019. Retrotransposon478 based genetic variation of Poa annua populations from contrasting climate conditions. PeerJ 7: 479 e6888 DOI 10.7717/peerj.6888.

480 Androsiuk P, Chwedorzewska KJ, Dulska J, Milarska S, Gielwanowska I. 2020.

481 Retrotransposon-based genetic diversity of Deschampsia antarctica Desv. From King George 482 Island (Maritime Antarctic). Ecology and Evolution 2020; 00:1-16 DOI 10.1002/ece3.7095.

483 Baránek M, Meszáros M, Sochorová J, Čechová J, Raddová J. 2012. Utility of retrotransposon 484 derived marker systems for differentiation of presumed clones of the apricot cultivar 485 Velkopavlovická. Scientia Horticulturae 143: 1-6 DOI 10.1016/j.scienta.2012.05.022.

486 Borna F, Luo S, Ahmad NM, Trathwan R. 2017. Genetic diversity in populations of the 487 medicinal plant Leonurus cardiaca L. revealed by inter-primer binding site (iPBS) markers. 488 Genetic Resources and Crop Evolution 64(3): 479-492 DOI 10.1007/s10722-016-0373-4.

489 Bradley D, Carpenter R, Sommer H, Hartley N, Coen E. 1993. Complementary floral homeotic 490 phenotypes result from opposite orientations of a transposon at the plena locus of Antirrhinum. 491 Cell 72(1): 85-95.

492 Chen FY, Liu JH. 2014. Germplasm genetic diversity of Myrica rubra in Zhejiang Province 493 studied using inter-primer binding site and start codon-targeted polymorphism markers. Scientia 494 Horticulturae 170: 169-175, DOI 10.1016/j.scienta.2014.03.010.

495 Earl DA, Vonholdt BM. 2012. STRUCTURE HARVESTER: a website and program for 496 visualizing STRUCTURE output and implementing the Evanno method. Conservation Genetics 497 Resources 4(2): 359-361, DOI 10.1007/s12686-011-9548-7.

498 Endo T. 1962. Inheritance of anthocyanin concentrations in flowers of Torenia fournieri. The 499 Japanese Journal of Genetics 37: 284-290.

500 Evanno G, Regnaut S, Goudet J. 2005. Detecting the number of clusters of individuals using the 501 software STRUCTURE: a simulation study. Molecular Ecology 14(8): 2611-2620, DOI

502 10.1111/j.1365-294X.2005.02553.x 
503 Gailite A, Rungis D. 2012. An initial investigation of the taxonomic status of Saussurea

504 esthonica Baer ex Rupr. Utilizing DNA markers and sequencing. Plant Systematics and

505 Evolution 298: 913-919.

506 Guo DL, Guo MX, Hou XG, Hang GH. 2014. Molecular diversity analysis of grape varieties

507 based on iPBS markers. Biochemical Systematics and Ecology 52: 27-32, DOI

508 10.1016/j.bse.2013.10.008.

509 Hess D. 1971. Chemogenetical investigations on the synthesis of flower colour substances in

510 Torenia species (Scrophulariaceae). Biochem. Physiol. Pflanz. 162: 386-389.

511 Higashiyama T, Yabe S, Sasaki N, Nishimura Y, Miyagishima S, Kuroiwa H, Kuroiwa T, 2001.

512 Pollen tube attraction by the synergid cell. Science 293: 1480-1483.

513 Higashiyama T, Kuroiwa H, Kawano S, Kuroiwa T. 1997. Kinetics of double fertilization in

514 Torenia fournieri based on direct observations of the naked embryo sac. Planta 203: 101-110.

515 Higashiyama T, Kuroiwa H, Kawano S, Kuroiwa T. 1998. Guidance in vitro of the pollen tube to 516 the naked embryo sac of Torenia fournieri. Plant Cell 10(12): 2019-2032.

517 Higashiyama T, Inatsugi R, Sakamoto S, Sasaki N, Mori T, Kuroiwa H, Nakada T, Nozaki H,

518 Kuroiwa T, Nakano A. 2006. Species preferentiality of the pollen tube attractant derived from

519 the synergid cell of Torenia fournieri. Plant Physiology 142(2): 481-491.

520 Hsieh TH, Yang KC. 2002. Revision of Torenia L. (Scophulariaceae) in Taiwan. Taiwania 521 47(4): 281-289 DOI 10.6165/tai.2002.47(4).281.

522 Iwaki K. 2005. U.S. Patent PP16055; U.S. Patent PP15560; U.S. Patent PP15684, Torenia plant 523 named 'Sunrenilapa'; 'Sunrenilahopas'; 'Sunrenilamu'. United State Patent \& Trademark Office.

524 Kalendar R, Amenov A, Daniyarov A. 2019. Use of retrotransposon-derived genetic markers to 525 analyse genomic variability in plants. Functional Plant Biology 46: 15-29 DOI

526 10.1071/FP18098.

527 Kalendar R, Antonius K, Smýkal P, Schulman AH. 2010. iPBS: a universal method for DNA 528 fingerprinting and retrotransposon isolation. Theoretical and Applied Genetics 121(8): 1419529 1430, DOI 10.1007/s00122-010-1398-2.

530 Keijzer CJ, Reinders MC, Leferink -ten KHB.1988. A micromanipulation method for artificial 531 fertilization in Torenia, p.119-124. In: Cresti M., Gori P, Pacini E. (eds) Sexual reproduction in 532 higher plants. Springer, Berlin, Heidelberg DOI 10.1007/978-3-642-73271-3_19.

533 Kikuchi S, Kishii M, Shimizu M, Tsujimoto H. 2005. Centromere-specific repetitive sequences 534 from Torenia, a model plant for interspecific fertilization, and whole-mount FISH of its 535 interspecific hybrid embryos. Cytogenetic and Genome Research 109: 228-235. 
536 Kikuchi S, Kino H, Tanaka H, Tsujimoto H. 2007a. Pollen tube growth in cross combinations

537 between Torenia fournieri and fourteen related species. Breeding Science 57: 117-122.

538 Kikuchi S, Tanaka H, Wako T, Tsujimoto H. 2007b. Centromere separation and association in

539 the nuclei of an interspecific hybrid between Torenia fournieri and T. baillonii

540 (Scrophulariaceae) during mitosis and meiosis. Genes \& Genetic Systems 82: 369-375.

541 Kikuchi S, Tanaka H, Shiba T, Mill M, Tsujimoto H. 2006. Genome size, karyotype, meiosis and

542 a novel extra chromosome in Torenia fournieri, T. baillonii and their hybrid. Chromosome

543 Research 14: 665-672.

544 Kumar S, Stecher G, Tamura K. 2016. MEGA7: molecular evolutionary genetics analysis

545 version 7.0 for bigger datasets. Molecular Biology and Evolution 33(7): 1870-1874 DOI

$546 \quad 10.1093 / \mathrm{molbev} / \mathrm{msw} 054$.

547 Lang W. 1970. Carotenoids of petals of Torenia baillonii and Torenia fournieri

548 (Scrophulariaceae). Z. Pflazenphysiol. 62: 299-301.

549 Laojunta T, Narumi-Kawasaki T, Takamura T, Fukai S. 2019. A new interspecific hybrid

550 of Torenia obtained through ovule culture. Horticulture Environment and Biotechnology 60:

$551 \quad 443-452$.

552 Liang ZC. 2014. Selection and Mutation Breeding of Interspecific Polyploid Torenia Hybrid.

553 Master's degree Thesis. Department of Horticulture, Taiwan University DOI

554 10.6342/NTU.2014.01204.

555 Luo S, Goikoetxea Arango A, Mehmood A, Ahmad N, Brown G. 2015. Developing New

556 Cordylines via Interspecific Hybridisation of the Australian Native Cordyline stricta with the

557 New Zealand Natives C. australis and the Cultivar 'Red Fountain' (C. hybrida). Acta

558 Horticulturae 1097: 205-212.

559 Masahiro O, Mitsuyo I, Phithak I, Kanyaratt S, Shiro M, Tomoya N, Takaaki N, Masaji K,

560 Masaru N. 2014. Horticultural characterization of a tetraploid transgenic plant of Tricyrtis sp.

561 carrying the gibberellin 2-oxidase gene. Plant Biotechnology 31(4): 335-340 DOI

$56210.5511 /$ plantbiotechnology.14.0916a.

563 Mehmood A, Jaskani MJ, Khan IA, Ahmad S, Ahmad R, Luo S, Ahmad NM. 2014. Genetic

564 diversity of Pakistani guava (Psidium guajava L.) germplasm and its implications for

565 conservation and breeding. Scientia Horticulturae 172: 221-232.

566 Mehmood A, Luo S, Ahmad N, Jaskani MJ, Sharp P. 2016. Molecular variability and

567 phylogenetic relationships of guava (Psidium guajava L.) cultivars using inter-primer binding

568 site (iPBS) and microsatellite (SSR) markers. Genetic Resources and Crop Evolution 63(8):

$569 \quad 1345-1361$. 
570 Miyazaki K. 1999. U.S. Patent PP11131: Torenia plant named 'Senrenidibu'. United State Patent

571 \& Trademark Office.

572 Miyazaki K. 2001. U.S. Patent PP12105: Torenia plant named 'Sunrenilabu'. United State Patent 573 \& Trademark Office.

574 Miyazaki K. 2002. Torenia plant named 'Sunrenihopas'. Plant Variety Protection, Ministry Agri. 575 For. Fish., Japan.

576 Miyazaki K. 2003. U.S. Patent PP14302: Torenia plant named 'Sunrenirirepa'. United State 577 Patent \& Trademark Office.

578 Miyazaki K, Ohsumi T. 1997. U.S. Patent PP10118: Torenia plant named 'Sunrenipiho'. United 579 State Patent \& Trademark Office.

580 Miyazaki K, Suzuki K, Iwaki K, Kusumi T, Abe T, Yoshida S, Fukui H. 2006. Flower pigment 581 mutations induced by heavy ion beam irradiation in an interspecific hybrid of Torenia. Plant 582 Biotechnology 23: 163-167.

583 Nagase Y. 1997. U.S. Patent PP10119: Torenia plant named 'Sunrenibu'. United State Patent \& 584 Trademark Office.

585 Nakamura N, Fukuchi-Mizutani M, Miyazaki K, Suzuki K, Tanaka Y. 2006. RNAi suppression 586 of the anthocyanidin synthase gene in Torenia hybrid yields white flowers with higher frequency 587 and better stability than antisense and sense suppression. Plant Biotechnology 23: 13-17.

588 Nakamura N, Fukuchi-Mizutani M, Fukui Y, Ishiguro K, Suzuki K, Suzuki H, Okazaki K, 589 Shibata D, Tanaka Y. 2010. Generation of pink flower varieties from blue Torenia hybrid by 590 redirecting the flavonoid biosynthetic pathway from delphinidin to pelargonidin. Plant 591 Biotechnology 27: 375-383.

592 Narumi T, Aida R, Koyama T, Yamaguchi H, Sasaki K, Shikata M, Nakayama M, Ohme-Takagi 593 M, Ohtsubo N. 2011. Arabidopsis chimeric TCP3 repressor produces novel floral traits in 594 Torenia fournieri and Chrysanthemum morifolium. Plant Biotechnology 28: 131-140.

595 Niki T, Hirai M, Niki T, Kanno A, Nishijima T. 2012. Role of floral homeotic genes in the 596 morphology of forchlorfenuron-induced paracorollas in Torenia fournieri Lind. Journal of the

597 Japanese Society for Horticultural Science 81: 204-212.

598 Niki T, Aida R, Niki T, Nishijima T. 2013a. Effect of localized promotion of cytokinin 599 biosynthesis on flower morphology in flower buds of Torenia fournieri Lind. Journal of the 600 Japanese Society for Horticultural Science 82: 328-336.

601 Niki T, Mahesumu T, Niki T, Nishijima T. 2013b. Localized high expression of type-A response 602 regular and cytokinin oxidase/dehydrogenase genes in relation to forchlorfenuron-induced 
603 changes in flower morphology in Torenia fournieri Lind. Journal of the Japanese Society for

604 Horticultural Science 82: 69-77.

605 Nishihara M, Shimoda T, Nakatsuka T, Arimura G. 2013. Frontiers of torenia research:

606 Innovative ornamental traits and study of ecological interaction networks through genetic

607 engineering. Plant Methods 9: 23-24.

608 Nishihara M, Higuchi A, Watanabe A, Tasaki K. 2018. Application of the CRISPR/Cas9 system

609 for modification of flower color in Torenia fournieri. BMC Plant Biology 18: 331 DOI

$610 \quad 10.1186 / \mathrm{s} 12870-018-1539-3$.

611 Nitasaka E. 2003. Insertion of an En/Spm-related transposable element into a floral homeotic

612 gene DUPLICATED causes a double flower phenotype in the Japanses moring glory. Plant

613 Journal 36: 522-531.

614 Nuntha B, Kikuchi S, Taychasinpitak T, Sassa H, Koba T. 2016. New karyotypes of an

615 interspecific hybrid of Torenia fournieri and Torenia baillonii and its progenies. Chromosome

616 Science 19: 37-40.

617 Nuntha B, Kikuchi S, Taychasinpitak T, Sassa H, Koba T. 2017. High genomic affinity between

618 Torenia baillonii and Torenia fournieri revealed by genome analysis using a triploid hybrid.

619 Cytologia 82(2): 213-218.

620 OGTR. 2008. The biology of Torenia spp. (Torenia). Office of the Gene Technology Regulator,

621 Department of Health and Ageing, Australian Government.

622 Okuda S, Tsutsui H, Shiina K, Sprunck S, Takeuchi H, Yui R, Kasahara RD, Hamamura Y, 623 Mizukami A, Susaki D, Kawano N, Sakakibara T, Namiki S, Itoh K, Otsuka K, Matsuzaki M, 624 Nozaki H, Kuroiwa T, Nakano A, Kanaoka MM, Dresselhaus T, Sasaki N, Higashiyama T.

625 2009. Defensin-like polypeptide LUREs are pollen tube attractants secreted from synergid cells.

626 Nature 458: 357-362.

627 Peakall R, Smouse PE. 2012. GenAlEx 6.5: genetic analysis in Excel. Population genetic

628 software for teaching and research - an update. Bioinformatics 28(19): 2537-2539 DOI

629 10.1093/bioinformatics/bts460.

630 Pritchard JK, Stephens P, Donnelly P. 2000. Inference of population structure using multi-locus 631 genotype data. Genetics 155: 945-959.

632 Sasaki K, Ohtsubo N. 2020. Production of multi-petaled Torenia fournieri flowers by functional

633 disruption of two class-C MADS-box genes. Planta 251: 101 DOI 10.1007/s00425-020-03393-3

634 Singha S, Nandhaa PS, Singha J. 2017. Transposon-based genetic diversity assessment in wild

635 and cultivated barley. Crop Journal 5: 296-304 DOI 10.1016/j.cj.2017.01.003.

636 Sneath PHA, Sokal RR. 1973. Numerical Taxonomy. Freeman, San Francisco. 
637 Suzuki K, Xui HM, Tanaka Y, Fukui Y, Fukuchi-Mizutani M, Murakami Y, Katsumoto Y,

638 Tsuda S, Kusumi T. 2000. Flower color modification of Torenia hybrid by cosupression of

639 anthocyanin biosynthesis genes. Molecular Breeding 6: 239-246.

640 Suzuki K, Miyazaki K, Tanaka Y, Iwaki K. 2002. Torenia plant named 'Sunreniho';

641 'Sunrenibuho'. Plant Variety Protection, Ministry Agric. For. Fish. Japan.

642 Tamura K, Nei M, Kumar S. 2004. Prospects for inferring very large phylogenies by using the

643 neighbor-joining method. Proceedings of the National Academy of Sciences of the United States

644 of America 101: 11030-11035.

645 Tamura Y, Miyazaki K. 1999. U.S. Patent PP10843: Torenia plant named 'Sunrenilabu'. United

646 State Patent \& Trademark Office.

647 Tandon SL, Bhutani K. 1965. Morphological and cytological studies of colchicines-induced

648 tetraploids in Torenia fournieri Lind. Genetica 36: 439-445.

649 Ueyama Y, Suzuki K, Fukuchi-Mizutani M, Fukui Y, Miyazaki K, Ohkawa H, Kusumi T,

650 Tanaka Y. 2002. Molecular and biochemical characterization of Torenia flavonoid 3'-

651 hydroxylase and flavone synthase II and modification of flower color by modulating the

652 expression of these genes. Plant Science 163: 253-263.

653 Wei TY. 2010. Inheritance of flower characteristics and distant hybridization barriers of

654 Torenias. Master's degree Thesis. Department of Horticulture, Taiwan University DOI

655 10.6342/NTU.2010.03262.

$656 \mathrm{Wu}$ J, Qin Y, Zhao J. 2008a. Pollen tube growth is affected by exogenous hormones and 657 correlated with hormone changes in styles in Torenia fournieri L. Plant Growth Regulation 55:

658 137-148.

659 Wu J, Lin Y, Zhang XL, Pang DW, Zhao J. 2008b. IAA stimulates pollen tube growth and 660 mediates the modification of its wall composition and structure in Torenia fournieri. Journal of 661 Experimental Botany 59: 2529-2543.

662 Yamazaki T. 1985. A revision of the genera Limnophila and Torenia from Indochina. Journal of 663 Faculty of Science, University of Tokyo Section III 13: 575-624.

664 Zhou J, Guan S, Song Q, Yan H, Zhang Z, Sun M, Pan Y, Luo S. 2021. Retrotransposon-based 665 genetic variation and population structure of Impatiens macrovexilla Y. L. Chen in natural 666 habitats and the implications for breeding. Scientia Horticulturae 276: 109753 DOI

667 10.1016/j.scienta.2020.109753. 


\section{Table $\mathbf{1}$ (on next page)}

Seven iPBS primers used in the detection of polymorphism among 136 accessions from 17 Torenia lines/populations.

A total of 136 accessions for 17 Torenia lines/populations included Duchess series ( 3 population of 24 accessions), Kauai series (7 population of 56 accessions), Little Kiss series (5 population of 40 accessions) and wild population ( 2 population of 16 accessions). $*$ Total accountable bands consistently appearing in two or three repeated experiments. ${ }^{1} \mathrm{PIC}$ value is calculated as PIC $=1-\left[f^{2}+(1-f)^{2}\right]$, where $f$ is the frequency of the dominant marker in the data set. 
1 Table 1:

2 Seven iPBS primers used in the detection of polymorphism among 136 accessions from 17 Torenia lines/populations.

3

\begin{tabular}{|c|c|c|c|c|c|c|c|c|}
\hline iPBS primer & Sequence (5'-3') & $\mathrm{Ta}^{\circ} \mathrm{C}$ & Number of bands* & Duchess Series & Kauai Series & Little Kiss Series & wild population & Mean PIC value ${ }^{1}$ \\
\hline 2076 & GCTCCGATGCCA & 39 & 21 & 12 & 17 & 14 & 15 & 0.249 \\
\hline 2077 & CTCACGATGCCA & 39 & 19 & 12 & 14 & 14 & 15 & 0.220 \\
\hline 2272 & GGCTCAGATGCCA & 39 & 20 & 15 & 15 & 14 & 15 & 0.291 \\
\hline 2277 & GGCGATGATACCA & 43.5 & 28 & 22 & 24 & 19 & 18 & 0.327 \\
\hline 2377 & ACGAAGGGACCA & 43.5 & 16 & 10 & 11 & 11 & 12 & 0.246 \\
\hline 2387 & GCGCCATACCCA & 39 & 20 & 14 & 16 & 13 & 15 & 0.300 \\
\hline 2383 & GCATGGCCTCCA & 43.5 & 24 & 14 & 16 & 14 & 18 & 0.239 \\
\hline Total & & & 148 & 99 & 113 & 99 & 108 & 0.267 \\
\hline
\end{tabular}

4 A total of 136 accessions for 17 Torenia lines/populations included Duchess series (3 population of 24 accessions), Kauai series (7 population of 56 accessions), Little Kiss series

5 (5 population of 40 accessions) and wild population ( 2 population of 16 accessions). ${ }^{*}$ Total accountable bands consistently appearing in two or three repeated experiments. ${ }^{1}$ PIC 6 value is calculated as $\mathrm{PIC}=1-\left[\mathrm{f}^{2}+(1-\mathrm{f})^{2}\right]$, where $\mathrm{f}$ is the frequency of the dominant marker in the data set. 


\section{Table 2 (on next page)}

Summary statistics of 136 Torenia accessions from 17 lines/populations evaluated with 7 iPBS primers.

$\mathrm{N}$, number of sample size; Na, number of different alleles; Ne, number of effective alleles $=1 /\left(p^{\wedge} 2+q^{\wedge} 2\right)$; I, Shannon's information index $=-1 *(p * \operatorname{Ln}(p)+q * \operatorname{Ln}(q))$; He, expected heterozygosity $=2 * p * q ; u H e$, unbiased expected heterozygosity $=(2 \mathrm{~N} /(2 \mathrm{~N}-1)) *$ He. Where for diploid binary data and assuming HardyWeinberg Equilibrium, $q=(1-\text { Band Freq. })^{\wedge} 0.5$ and $p=1-q$.

A: Duchess Pink; B: Duchess Burgundy; C: Duchess Deep Blue; D: Kauai Burgundy; E: Kauai Rose; F: Kauai Deep Blue; G: Kauai Blue and White; H: Kauai Magenta; I: Kauai Lemon Drop; J: Kauai White; K: Little Kiss White; L: Little Kiss Burgundy; M: Little Kiss Blue and White; N: Little Kiss Rose Picotee; P: Little Kiss Blue; Q: Lipu; R: Xichou. 
1 Table 2:

2 Summary statistics of 136 Torenia accessions from 17 lines/populations evaluated with 7 iPBS primers.

\begin{tabular}{|c|c|c|c|c|c|c|c|}
\hline Populations & $\mathrm{N}$ & $\mathrm{Na}$ & $\mathrm{Ne}$ & I & $\mathrm{He}$ & $\mathrm{uHe}$ & Polymorphic loci\% \\
\hline A & 8 & $0.7500 \pm 0.0598$ & $1.1101 \pm 0.0223$ & $0.0928 \pm 0.0178$ & $0.0629 \pm 0.0123$ & $0.0671 \pm 0.0131$ & $16.89 \%$ \\
\hline B & 8 & $0.6757 \pm 0.0518$ & $1.0514 \pm 0.0153$ & $0.0458 \pm 0.0127$ & $0.0305 \pm 0.0087$ & $0.0325 \pm 0.0093$ & $8.78 \%$ \\
\hline $\mathrm{C}$ & 8 & $0.6351 \pm 0.0481$ & $1.0302 \pm 0.0121$ & $0.0269 \pm 0.0099$ & $0.0178 \pm 0.0067$ & $0.0190 \pm 0.0072$ & $5.41 \%$ \\
\hline $\mathrm{D}$ & 8 & $0.6892 \pm 0.0524$ & $1.0567 \pm 0.0168$ & $0.0486 \pm 0.0132$ & $0.0325 \pm 0.0091$ & $0.0346 \pm 0.0097$ & $9.46 \%$ \\
\hline $\mathrm{E}$ & 8 & $0.7568 \pm 0.0564$ & $1.0894 \pm 0.0202$ & $0.0770 \pm 0.0163$ & $0.0518 \pm 0.0112$ & $0.0553 \pm 0.0119$ & $14.19 \%$ \\
\hline $\mathrm{F}$ & 8 & $0.7365 \pm 0.0609$ & $1.1196 \pm 0.0236$ & $0.0990 \pm 0.0184$ & $0.0675 \pm 0.0128$ & $0.0720 \pm 0.0136$ & $17.57 \%$ \\
\hline G & 8 & $0.7027 \pm 0.0595$ & $1.0973 \pm 0.0208$ & $0.0842 \pm 0.0169$ & $0.0566 \pm 0.0116$ & $0.0604 \pm 0.0124$ & $15.54 \%$ \\
\hline $\mathrm{H}$ & 8 & $0.7365 \pm 0.0609$ & $1.1305 \pm 0.0248$ & $0.1047 \pm 0.0192$ & $0.0723 \pm 0.0134$ & $0.0772 \pm 0.0143$ & $17.57 \%$ \\
\hline I & 8 & $0.5338 \pm 0.0493$ & $1.0309 \pm 0.0122$ & $0.0274 \pm 0.0100$ & $0.0182 \pm 0.0069$ & $0.0194 \pm 0.0073$ & $5.41 \%$ \\
\hline $\mathrm{J}$ & 8 & $0.4865 \pm 0.0465$ & $1.0243 \pm 0.0112$ & $0.0200 \pm 0.0090$ & $0.0138 \pm 0.0062$ & $0.0147 \pm 0.0066$ & $3.38 \%$ \\
\hline K & 8 & $0.5473 \pm 0.0528$ & $1.0464 \pm 0.0149$ & $0.0409 \pm 0.0121$ & $0.0271 \pm 0.0083$ & $0.0290 \pm 0.0088$ & $8.11 \%$ \\
\hline $\mathrm{L}$ & 8 & $0.6216 \pm 0.0562$ & $1.0600 \pm 0.0164$ & $0.0549 \pm 0.0135$ & $0.0360 \pm 0.0092$ & $0.0383 \pm 0.0098$ & $11.49 \%$ \\
\hline M & 8 & $0.6081 \pm 0.0611$ & $1.1151 \pm 0.0234$ & $0.0934 \pm 0.0182$ & $0.0644 \pm 0.0127$ & $0.0686 \pm 0.0135$ & $15.54 \%$ \\
\hline $\mathrm{N}$ & 8 & $0.4932 \pm 0.0530$ & $1.0609 \pm 0.0186$ & $0.0472 \pm 0.0137$ & $0.0327 \pm 0.0097$ & $0.0349 \pm 0.0103$ & $8.11 \%$ \\
\hline$P$ & 8 & $0.6284 \pm 0.0616$ & $1.1111 \pm 0.0232$ & $0.0908 \pm 0.0178$ & $0.0620 \pm 0.0125$ & $0.0662 \pm 0.0133$ & $16.22 \%$ \\
\hline Q & 8 & $0.6014 \pm 0.0619$ & $1.1067 \pm 0.0222$ & $0.0900 \pm 0.0175$ & $0.0609 \pm 0.0121$ & $0.0650 \pm 0.0129$ & $16.22 \%$ \\
\hline $\mathrm{R}$ & 8 & $0.9932 \pm 0.0788$ & $1.2885 \pm 0.0309$ & $0.2453 \pm 0.0239$ & $0.1655 \pm 0.0167$ & $0.1766 \pm 0.0178$ & $45.27 \%$ \\
\hline Mean & 8 & $0.6586 \pm 0.0141$ & $1.0899 \pm 0.0050$ & $0.0758 \pm 0.0039$ & $0.0513 \pm 0.0027$ & $0.0547 \pm 0.0029$ & $13.83 \% \pm 2.28 \%$ \\
\hline
\end{tabular}


$4 \mathrm{~N}$, number of sample size; Na, number of different alleles; Ne, number of effective alleles $=1 /\left(p^{\wedge} 2+q^{\wedge} 2\right)$; I, Shannon’s information index $=-1^{*}(p * \operatorname{Ln}(p)+q * \operatorname{Ln}(q)) ; \operatorname{He}$, expected heterozygosity $=2 * \mathrm{p} * \mathrm{q}$; uHe, unbiased expected heterozygosity $=(2 \mathrm{~N} /(2 \mathrm{~N}-1)) *$ He. Where for diploid binary data and assuming Hardy-Weinberg Equilibrium, $\mathrm{q}=$ $(1 \text { - Band Freq. })^{\wedge} 0.5$ and $p=1$ - q.

7 A: Duchess Pink; B: Duchess Burgundy; C: Duchess Deep Blue; D: Kauai Burgundy; E: Kauai Rose; F: Kauai Deep Blue; G: Kauai Blue and White; H: Kauai Magenta; I: Kauai 8 Lemon Drop; J: Kauai White; K: Little Kiss White; L: Little Kiss Burgundy; M: Little Kiss Blue and White; N: Little Kiss Rose Picotee; P: Little Kiss Blue; Q: Lipu; R: Xichou. 


\section{Table 3 (on next page)}

Pairwise population PhiPT values for a total of 136 accessions from 17 Torenia lines/populations.

Pairwise population PhiPT values below diagonal. Values more than 0.895 marked as bold and values less than 0.600 are indicated by a subscript.

A: Duchess Pink; B: Duchess Burgundy; C: Duchess Deep Blue; D: Kauai Burgundy; E: Kauai Rose; F: Kauai Deep Blue; G: Kauai Blue and White; H: Kauai Magenta; I: Kauai Lemon Drop; J: Kauai White; K: Little Kiss White; L: Little Kiss Burgundy; M: Little Kiss Blue and White; N: Little Kiss Rose Picotee; P: Little Kiss Blue; Q: Lipu and R: Xichou. 


\section{Table 3:}

2 Pairwise population PhiPT values for a total of 136 accessions from 17 Torenia lines/populations.

3

\begin{tabular}{|c|c|c|c|c|c|c|c|c|c|c|c|c|c|c|c|c|c|}
\hline & $\mathrm{A}$ & B & $\mathrm{C}$ & $\mathrm{D}$ & $E$ & $F$ & $\mathrm{G}$ & $\mathrm{H}$ & I & $\mathrm{J}$ & $\mathrm{K}$ & $\mathrm{L}$ & $\mathrm{M}$ & $\mathrm{N}$ & $\mathrm{P}$ & $\mathrm{Q}$ & $\mathrm{R}$ \\
\hline $\mathrm{A}$ & 0.000 & & & & & & & & & & & & & & & & \\
\hline B & 0.700 & 0.000 & & & & & & & & & & & & & & & \\
\hline $\mathrm{C}$ & 0.640 & 0.839 & 0.000 & & & & & & & & & & & & & & \\
\hline $\mathrm{D}$ & 0.686 & 0.769 & 0.798 & 0.000 & & & & & & & & & & & & & \\
\hline $\mathrm{E}$ & $\underline{0.542}$ & 0.752 & 0.638 & 0.652 & 0.000 & & & & & & & & & & & & \\
\hline $\mathrm{F}$ & $\underline{0.563}$ & 0.722 & 0.702 & 0.652 & $\underline{0.581}$ & 0.000 & & & & & & & & & & & \\
\hline G & 0.700 & 0.779 & 0.800 & 0.802 & 0.738 & 0.699 & 0.000 & & & & & & & & & & \\
\hline $\mathrm{H}$ & 0.673 & 0.747 & 0.806 & 0.758 & 0.746 & 0.728 & 0.621 & 0.000 & & & & & & & & & \\
\hline I & 0.784 & 0.871 & 0.890 & 0.878 & 0.831 & 0.807 & 0.690 & 0.752 & 0.000 & & & & & & & & \\
\hline $\mathrm{J}$ & 0.754 & 0.852 & 0.895 & 0.875 & 0.809 & 0.757 & 0.740 & 0.765 & 0.830 & 0.000 & & & & & & & \\
\hline $\mathrm{K}$ & 0.751 & 0.855 & 0.878 & 0.865 & 0.806 & 0.779 & 0.710 & 0.708 & 0.771 & 0.801 & 0.000 & & & & & & \\
\hline $\mathrm{L}$ & 0.709 & 0.793 & 0.856 & 0.827 & 0.758 & 0.739 & 0.701 & 0.695 & 0.827 & 0.802 & 0.730 & 0.000 & & & & & \\
\hline M & 0.762 & 0.805 & 0.848 & 0.821 & 0.790 & 0.772 & 0.768 & 0.760 & 0.858 & 0.842 & 0.812 & 0.779 & 0.000 & & & & \\
\hline $\mathrm{N}$ & 0.829 & 0.877 & 0.913 & 0.878 & 0.840 & 0.835 & 0.835 & 0.813 & 0.917 & 0.912 & 0.878 & 0.843 & $\underline{0.588}$ & 0.000 & & & \\
\hline $\mathrm{P}$ & 0.767 & 0.817 & 0.851 & 0.827 & 0.796 & 0.784 & 0.765 & 0.749 & 0.851 & 0.838 & 0.808 & 0.783 & $\underline{0.336}$ & $\underline{0.562}$ & 0.000 & & \\
\hline Q & 0.849 & 0.880 & 0.901 & 0.878 & 0.854 & 0.841 & 0.843 & 0.848 & 0.898 & 0.904 & 0.887 & 0.863 & 0.797 & 0.842 & 0.806 & 0.000 & \\
\hline $\mathrm{R}$ & 0.758 & 0.770 & 0.810 & 0.777 & 0.778 & 0.746 & 0.750 & 0.725 & 0.779 & 0.791 & 0.768 & 0.752 & 0.716 & 0.759 & 0.705 & 0.754 & 0.000 \\
\hline
\end{tabular}

4 
5 Pairwise population PhiPT values below diagonal. Values more than 0.895 marked as bold and values less than 0.600 are indicated by a subscript. A: Duchess Pink; B: Duchess Burgundy; C: Duchess Deep Blue; D: Kauai Burgundy; E: Kauai Rose; F: Kauai Deep Blue; G: Kauai Blue and White; H: Kauai Magenta; I: Kauai Lemon Drop; J: Kauai White; K: Little Kiss White; L: Little Kiss Burgundy; M: Little Kiss Blue and White; N: Little Kiss Rose Picotee; P: Little Kiss Blue; Q: Lipu and R: Xichou. 


\section{Table 4 (on next page)}

Partitioning of genetic diversity for 17 (15) Torenia populations by AMOVA [ PhiPT = $0.794(0.140)]$.

Note: d.f. $=$ degree of freedom. Significance tests (999 permutations); $p<0.001$. The values in parentheses are values for the 15 cultivated lines. 
1 Table 4:

2 Partitioning of genetic diversity for 17 (15) Torenia populations by AMOVA [ PhiPT $=0.794(0.140)$ ].

\begin{tabular}{cccccc}
\hline Source of variation & d.f. & Sum of Square & Mean Squares & $\begin{array}{c}\text { Estimated } \\
\text { Variance }\end{array}$ & $\begin{array}{c}\text { Percentage of total } \\
\text { variation }\end{array}$ \\
\hline Among Populations & $16(16)$ & $2098.441(15.375)$ & $131.153(0.961)$ & $15.879(0.068)$ & $79 \%(14 \%)$ \\
Within Populations & $119(119)$ & $489.875(49.750)$ & $4.117(0.418)$ & $4.117(0.418)$ & $21 \%(86 \%)$ \\
$\quad$ Total & $135(135)$ & $2588.316(65.125)$ & & $19.996(0.486)$ & $100 \%(100 \%)$ \\
\hline
\end{tabular}

3

4 Note: d.f. $=$ degree of freedom. Significance tests (999 permutations); $p<0.001$. The values in parentheses are values for the 15 5 cultivated lines.

6 
Figure 1

Geographic location of the sampling sites for two wild Torenia concolor populations in southern China.

The map data is provided by Geospatial Data Cloud site, Computer Network Information

Center, Chinese Academy of Sciences. (http://www.gscloud.cn)

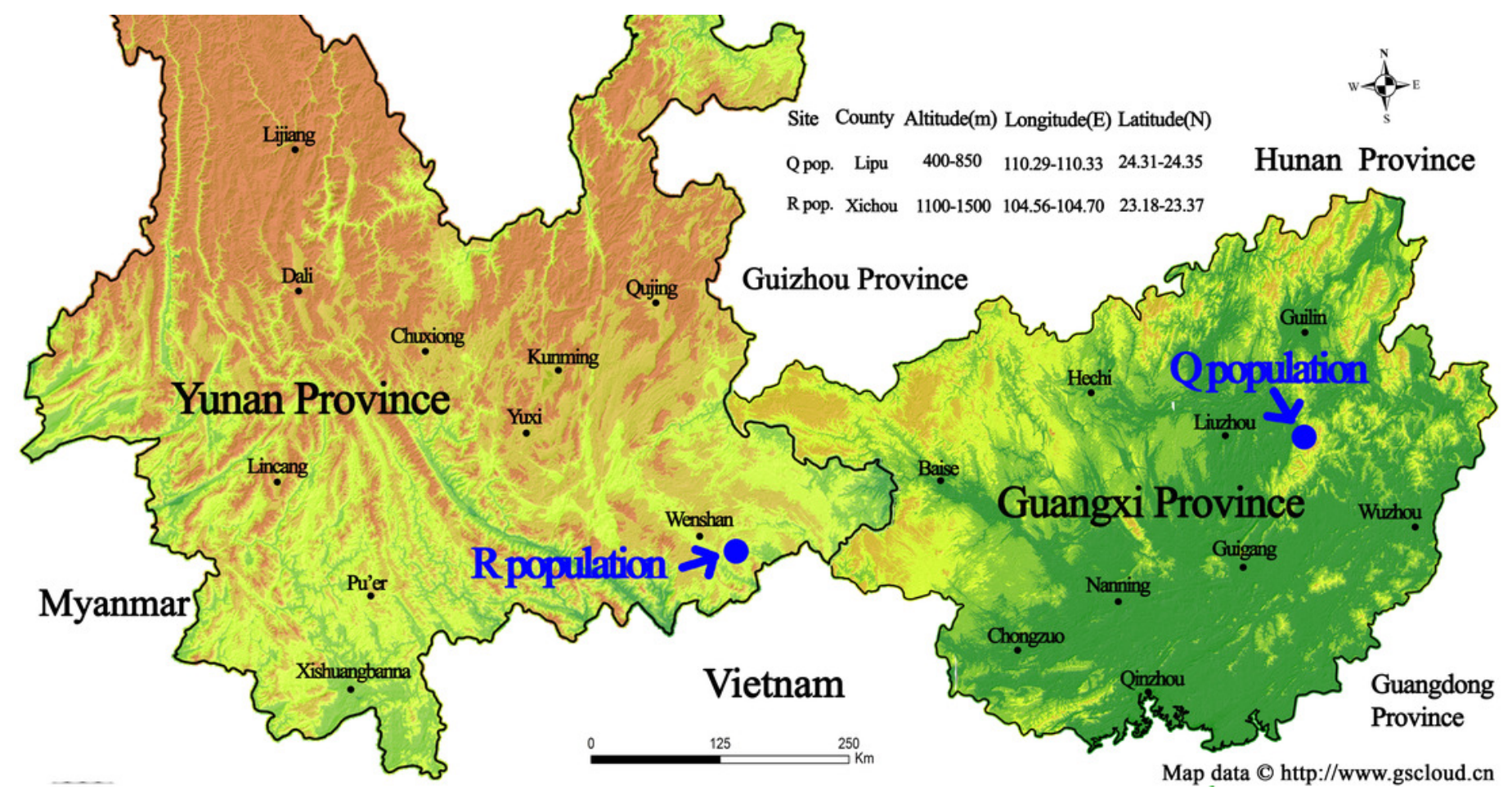




\section{Figure 2}

The floral colors for 17 Torenia lines/populations.

(A) Duchess Pink. (B) Duchess Burgundy. (C) Duchess Deep Blue. (D) Kauai Burgundy. (E) Kauai Rose. (F) Kauai Deep Blue. (G) Kauai Blue and White. (H) Kauai Magenta. (I) Kauai Lemon Drop. (J) Kauai White. (K) Little Kiss White. (L) Little Kiss Burgundy. (M) Little Kiss Blue and White. (N) Little Kiss Rose Picotee. (P) Little Kiss Blue. (Q) wild, Blue. (R) wild, Blue.

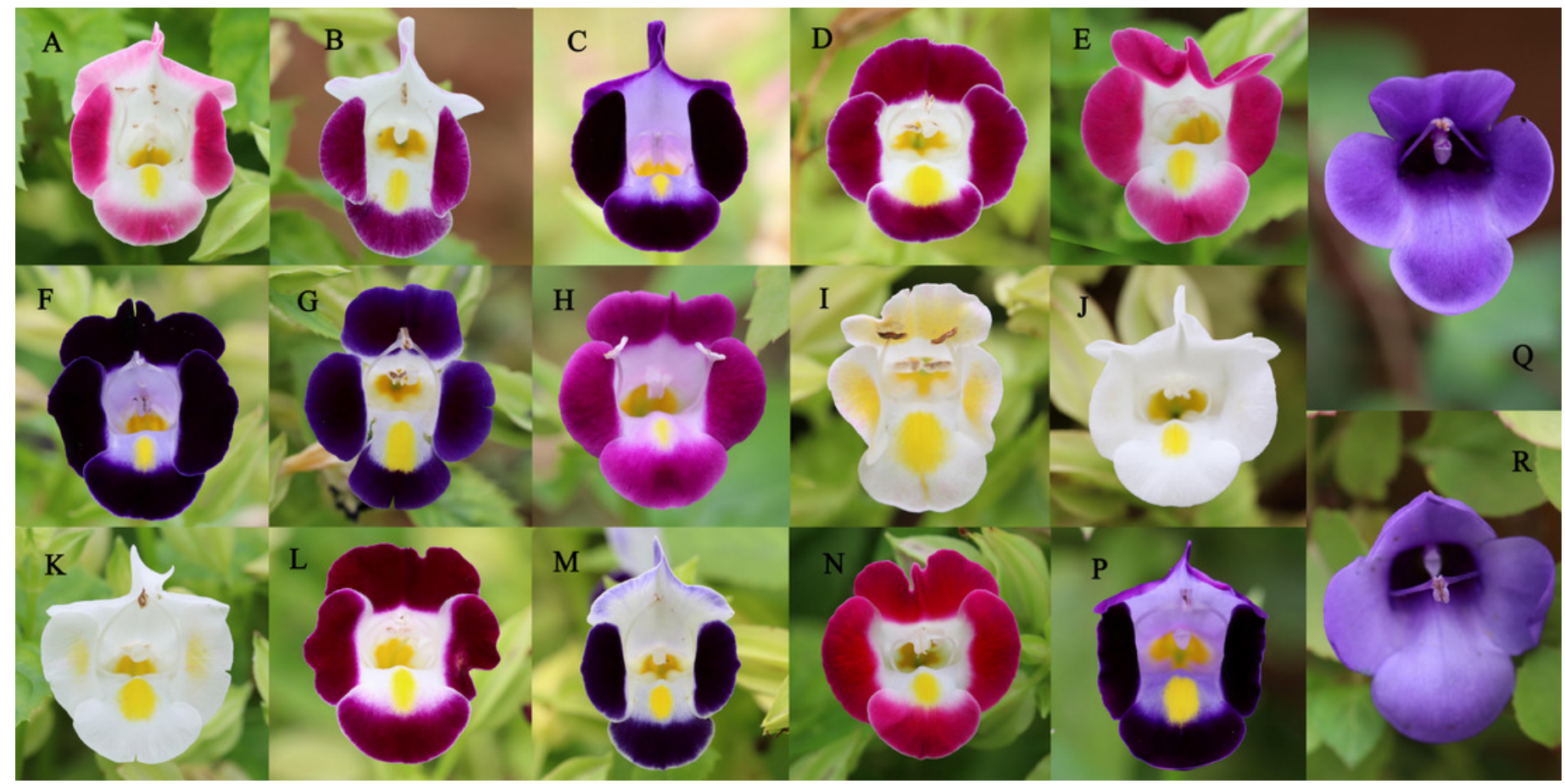


Figure 3

The plot of Coordinate 1 vs Coordinate 2 in principal coordinate analysis (PCOA) using all the Euclidean distances for the 136 individual accessions of 17 Torenia lines/populations.

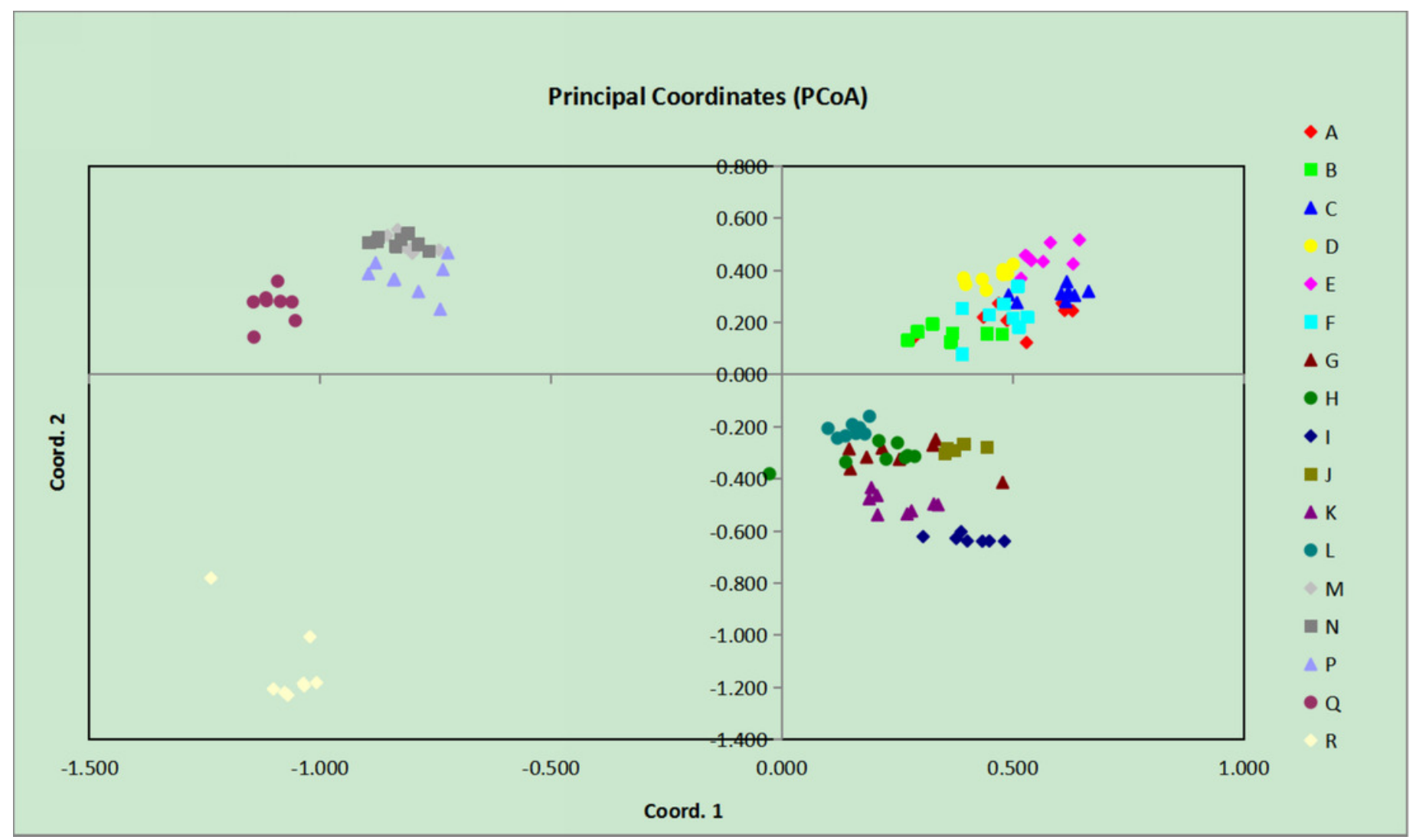




\section{Figure 4}

The evolutionary relationship of 136 Torenia taxa in 17 lines/populations derived from the combined data of seven iPBS primers.

A: Duchess Pink; B: Duchess Burgundy; C: Duchess Deep Blue; D: Kauai Burgundy; E: Kauai Rose; F: Kauai Deep Blue; G: Kauai Blue and White; H: Kauai Magenta; I: Kauai Lemon Drop; J: Kauai White; K: Little Kiss White; L: Little Kiss Burgundy; M: Little Kiss Blue and White; N: Little Kiss Rose Picotee; P: Little Kiss Blue; Q: wild, Blue; R: wild, Blue. 


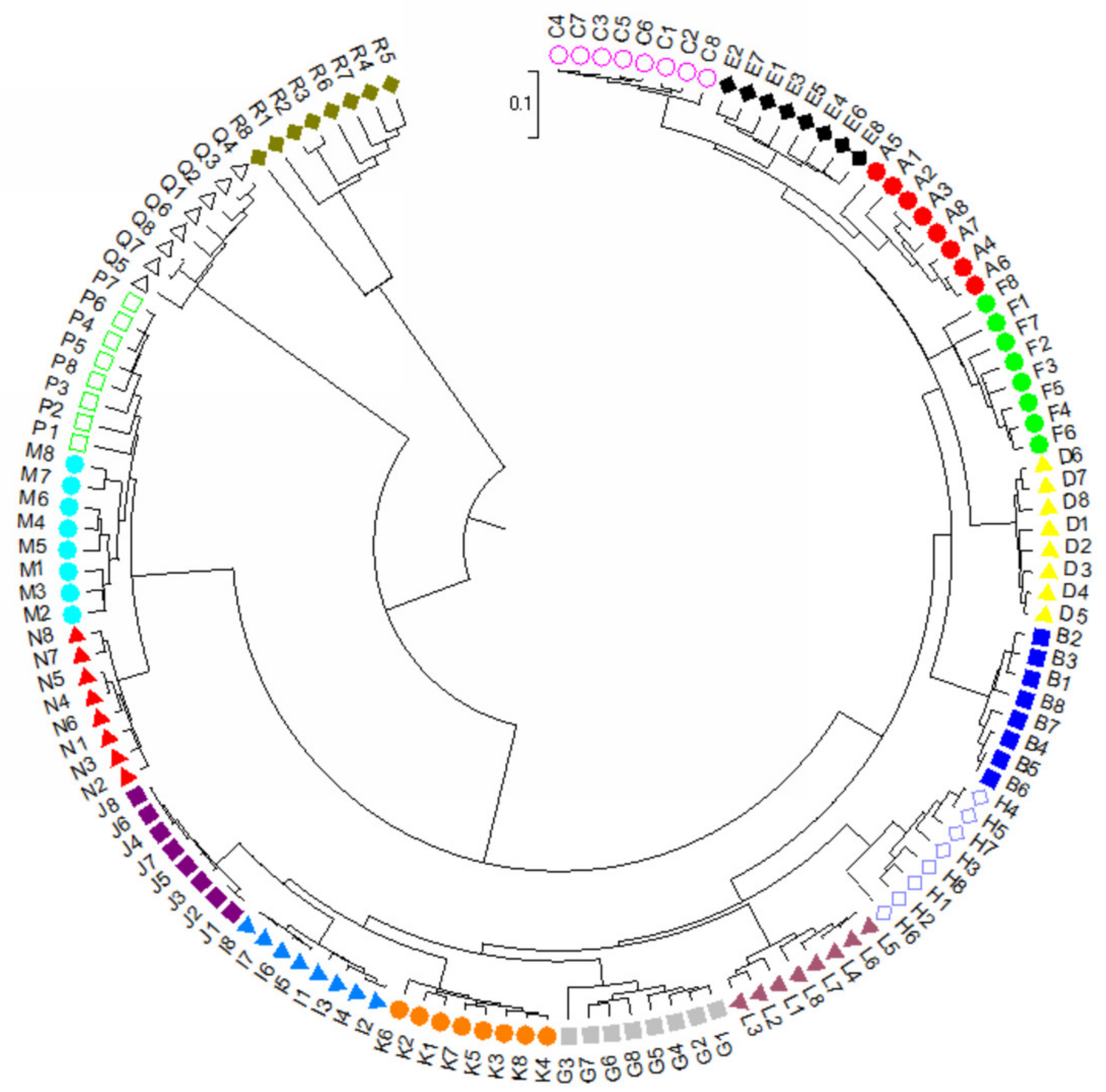


Figure 5

The final hierarchical level of genetic structure for 17 Torenia lines/populations using a Bayesian model-based cluster construction based on allelic variations at seven iPBS loci (Earl and Vonholdt, 2012).

Plot of the delta $\mathrm{K}$ (filled circles, solid line) according to the values of the second-order rate of change of $L(K), \Delta K$, and data between successive $K$ values.

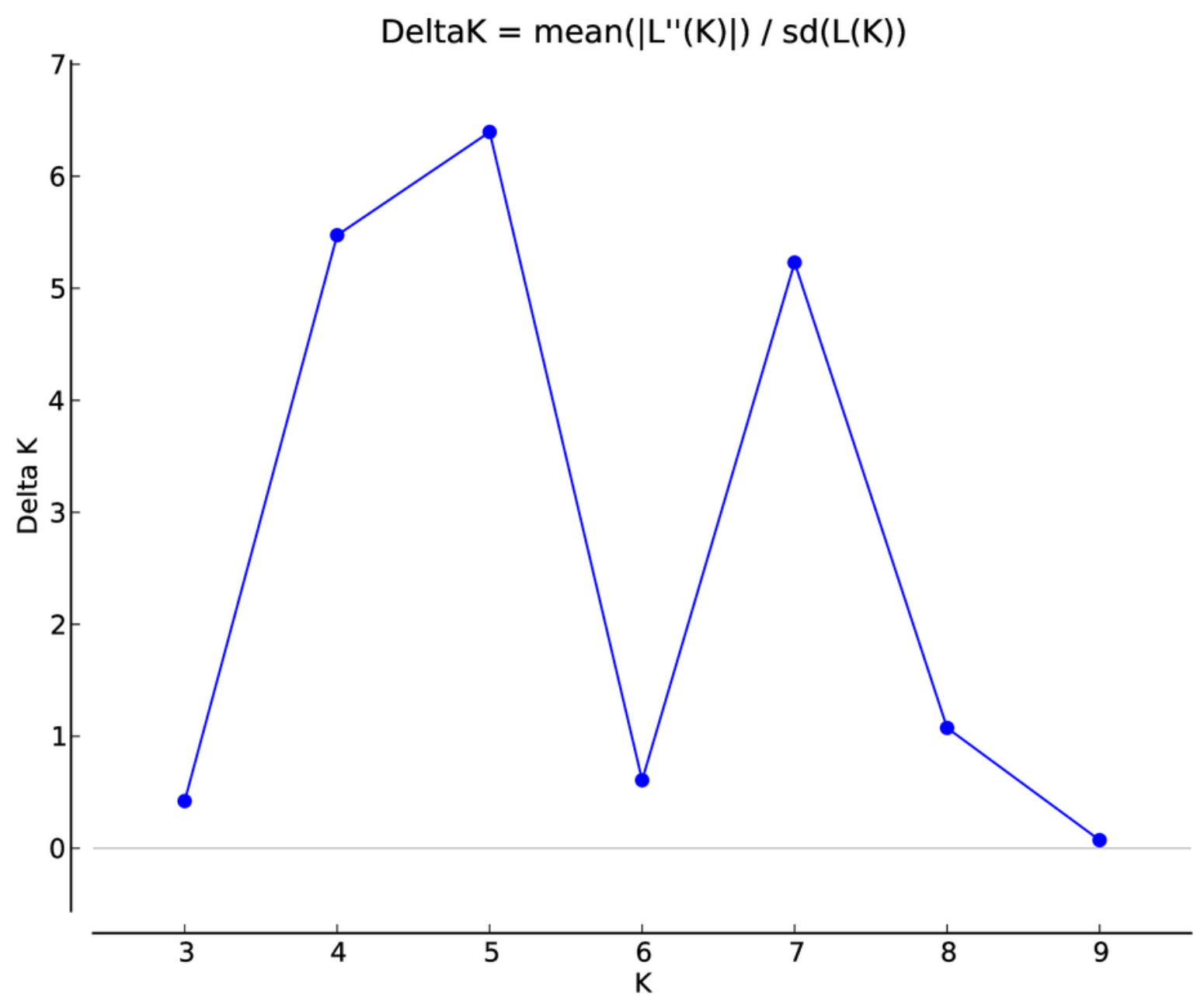




\section{Figure 6}

The final hierarchical level of genetic structure for 17 Torenia lines/populations using a Bayesian model-based cluster construction based on allelic variations at seven iPBS loci (Earl and Vonholdt, 2012).

Bar plots of the population genetic structure achieved at $\mathrm{K}=5$.
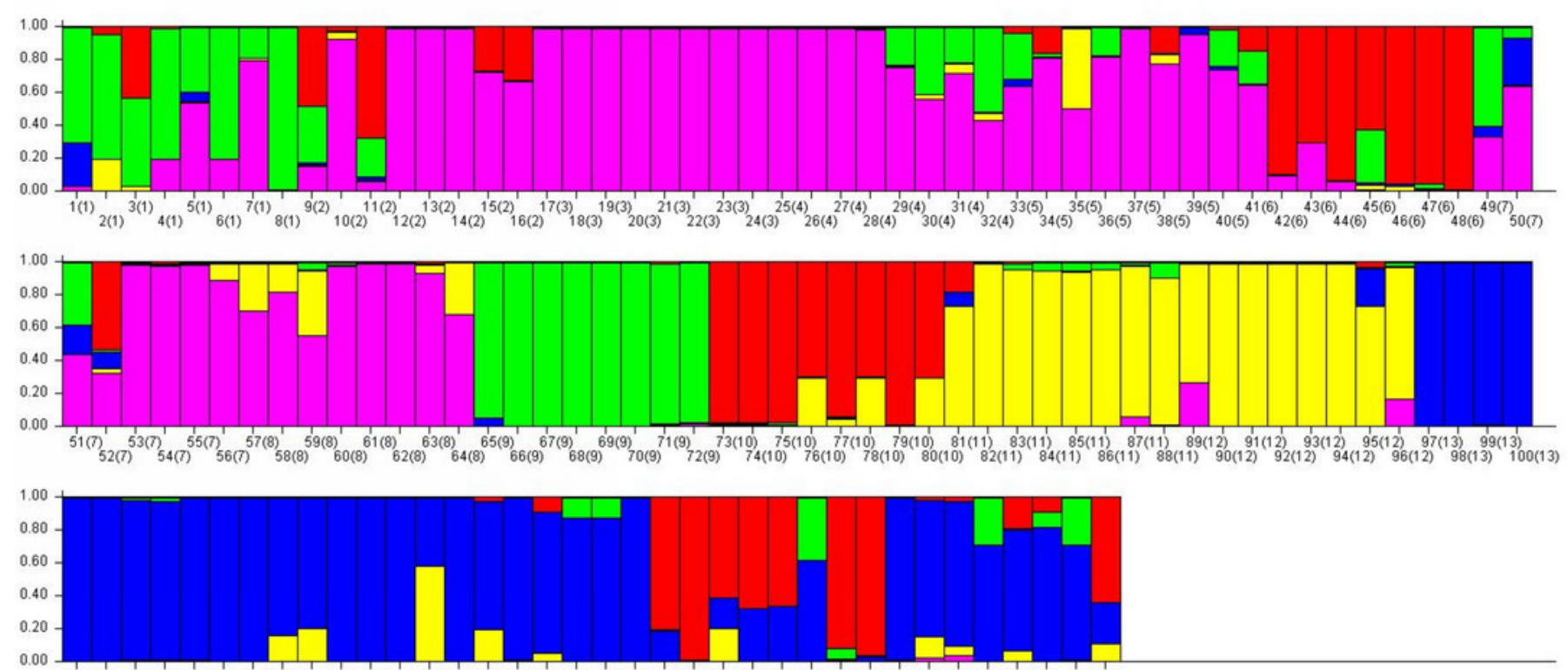